\title{
Imagining Adherence: Body Mapping Research with HIV-Positive Men and Women in Canada
}

\author{
Treena Orchard, ${ }^{1}$ Tricia Smith, ${ }^{2}$ Warren Michelow, ${ }^{3}$ Kate Salters, ${ }^{4}$ and Bob Hogg ${ }^{4}$
}

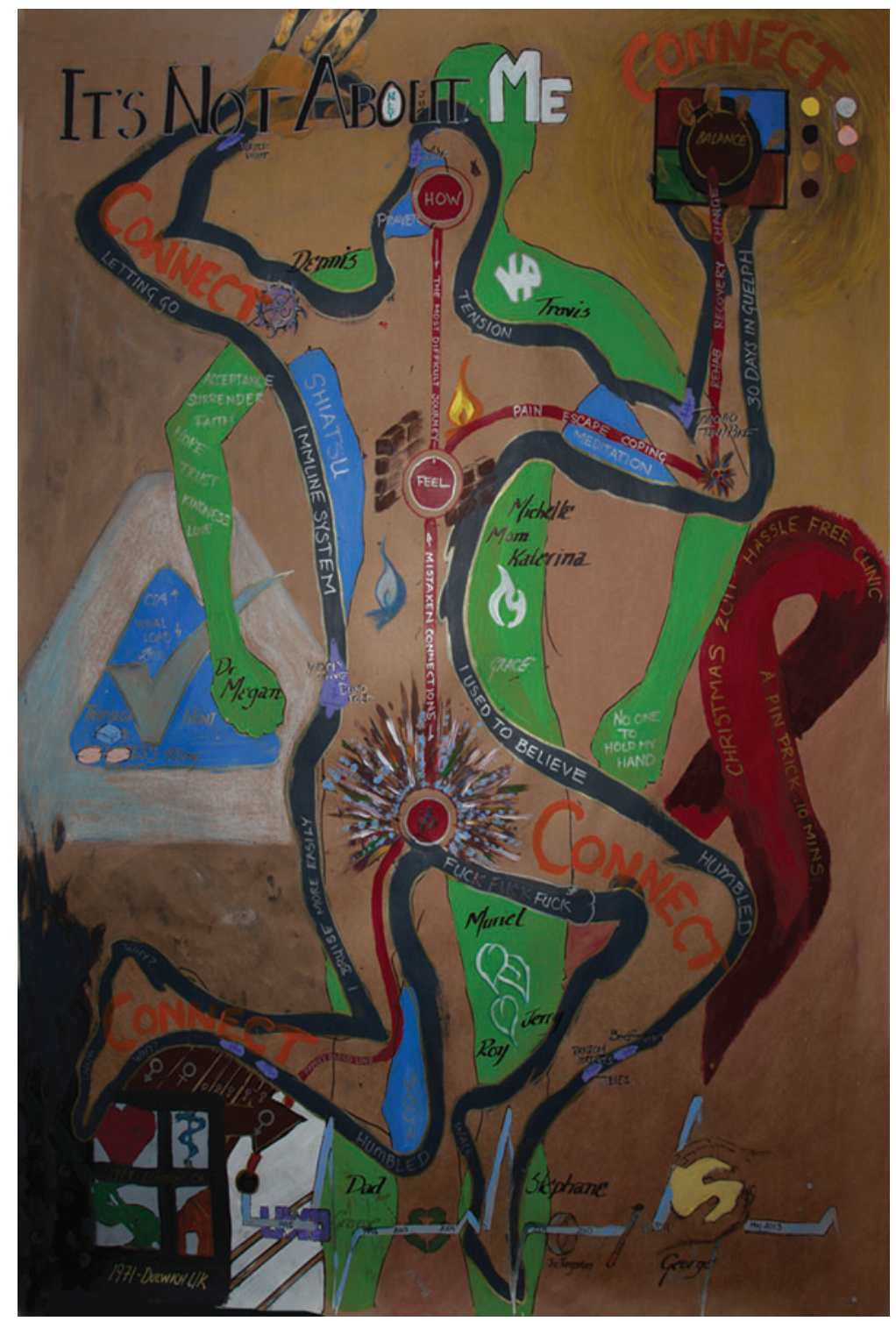

FIG. 1. Body map from a male participant.

\footnotetext{
${ }^{1}$ University of Western Ontario, London, Ontario, Canada.

${ }^{2}$ Queen's University, Kingston, Ontario, Canada.

${ }^{3}$ University of British Columbia, Vancouver, British Columbia, Canada.

${ }^{4} \mathrm{BC}$ Centre for Excellence in HIV/AIDS, Vancouver, British Columbia, Canada.
} 
T HE RELATIONSHIP BETWEEN artistic creativity and HIV/ AIDS has a long history, perhaps most notably during the early era of the epidemic when art was a vital tool in the expression of identity politics, the recognition of sexual and human rights, and the fight for equitable access to HIV/AIDS medications. Despite the critical role played by art and visual imagery during these years, comparatively little attention has been paid to the relationship between arts-based research and the HIV movement today. One exception to this is body mapping (Fig. 1). This arts-based approach was developed in South Africa as a way to combat HIV stigma, and involves the creation of life-sized maps that people use to express their HIV experiences and journeys. ${ }^{1}$ Originally envisioned as a way to capture the lives of those lost to HIV, body mapping in contemporary HIV research has also been used to examine key treatment-related issues that continue to evade biomedical research, including adherence to HIV medications

Most medical research on adherence to HIV medications examines the degree to which HIV-positive people follow prescribed medical advice. This approach does not fully consider how medical information is translated and incorporated into people's everyday health practices or how gender impacts medication practices. To address this research gap, we conducted individual interviews and body mapping workshops with men $(n=5)$ and women $(n=6)$ to explore how gender and ideas about the body impact HIV medication practices. This article features our body mapping data, gathered during two 4-day workshops led by a trained facilitator in two Canadian cities. Unlike biomedical approaches to adherence, which rest upon individualized models of health behavior and responsibility, for most of our participants adherence was positioned as a complex, situational, and culturally constructed set of practices. Informed by their accumulative HIV knowledge, these practices are also shaped by the active reading of their bodily responses to medications, resistance to biomedical modes of disease management, and personalized conceptions of what health means. A second unique research finding highlights how gender impacts medication practices, with women being more likely than men to take their medications regularly and to adopt the discourse of individual responsibility for the management of their HIV through the regular uptake of medications.

Another important project outcome relates to the specific attributes of the body mapping methodology, which our participants identified as a powerful conduit for the channeling of multilevel experiences and ideas related to self-care and self-actualization. Body mapping allowed them to direct the data creation and the visual communication of their lived experience in ways that are unique compared to surveys and individual interviews, which are often controlled by researchers and are dependent upon paper surveys or textual data. Our findings demonstrate that body mapping is a powerful and productive research methodology to illuminate a myriad of experiences and ideologies related to living with HIV that we are only beginning to fully understand.

\section{Author Disclosure Statement}

No competing financial interests exist.

\section{Reference}

1. Solomon J: Living with X: A Body Mapping Journal in the Time of HIV and AIDS: A Facilitator's Guide. 2007.

Address correspondence to:

Treena Orchard

University of Western Ontario School of Health Studies 208 Labatt Health Sciences Building London, Ontario N6A 5B9

Canada

E-mail: treena_orchard@yahoo.ca 